\title{
Demonstration of Self-Training Autonomous Neural Networks in Space Vehicle Docking Simulations
}

\author{
M. Clinton Patrick \\ NASA/MSFC/EV43 \\ Marshall Space Flight Center, AL 35812 \\ 256-544-3836 \\ clint.patrick@nasa.gov \\ Stephen L. Thaler \\ Imagination Engines, Inc., St. Louis, MO 63146 \\ 314-317-2228 \\ sthaler@imagination-engines.com \\ Katherine Stevenson-Chavis \\ NASA/MSFC/EV21 \\ Marshall Space Flight Center, AL 35812 \\ 256-544-6174 \\ katherine.m.chavis@nasa.gov
}

\begin{abstract}
Neural Networks have been under examination for decades in many areas of research, with varying degrees of success and acceptance. Key goals of computer learning, rapid problem solution, and automatic adaptation have been elusive at best. This paper ${ }^{1,2}$ summarizes efforts at NASA's Marshall Space Flight Center harnessing such technology to autonomous space vehicle docking for the purpose of evaluating applicability to future missions.
\end{abstract}

\section{TABLE OF CONTENTS}

1. INTRODUCTION . .1

2. BACKGROUND AND THEORY ..........................................2

3. EARLY RESULTS...............................................................

4. FIRST SERIES OF TESTS................................................

5. SECOND SERIES OF TESTS............................................... 4

6. PLANS FOR CONTINUED WORK .....................................5

7. CONCLUSIONS ...................................................................6

REFERENCES.....................................................................6

ACKNOWLEDGEMENTS..................................................6

BIOGRAPHIES...................................................................6

\section{INTRODUCTION}

One research laboratory at Marshall Space Flight Center (MSFC) is the Flight Robotics Lab. There, a particularly impressive piece of infrastructure is the Flat Floor, a large precision-poured epoxy surface designed to be planar to within about seven thousandths of an inch over large areas at a time. This allows levitation of very heavy test "sleds"

\footnotetext{
'U.S. Government work not protected by U.S. copyright.

${ }^{2}$ IEEEAC paper \# 1409, Version 1, Updated December 28, 2006
}

on the floor using air bearings fed from high pressure sources, these typically being contained on the test vehicle itself. Given such resources, nearly frictionless conditions can be produced for study of the motion of objects in three degrees of freedom. A prime use is found testing schemes for autonomous rendezvous and docking (AR\&D) in Space [1]. Figure 1 shows the Small Air Sled (SAS) on the Flat Floor.

Over the past few years, personnel at MSFC have been pursuing access to patented (e.g.: [3] through [10]) artificial neural network (ANN) technology developed by Imagination Engines, Inc. (IEI). A number of appealing target applications readily present themselves; however, AR\&D is perhaps the most appealing as a representation of NASA's core missions.



Figure 1: SAS and Target on the MSFC Flat Floor 
This work was made possible through the Independent Research and Development (IR\&D) program at MSFC [2]. Objectives of the research include demonstration of ANNs in performance of various AR\&D functions on the MSFC Flat Floor utilizing video camera inputs as a primary data source, realizing the networks in both software and hardware forms.

\section{BACKGROUND AND THEORY}

IEI's ANN technologies designated "Self-Training Autonomous Neural Network Objects" (STANNOs) and "Creativity Machines" have already been developed to a high level of capability through many years of experimentation. Implementation for specific tasks is often accomplished in minutes or hours, building upon a set of tools and experience previously groomed and tested in various other operations.

One major strength of STANNOs is ability to build, in software form, extremely large, multilayer ANNs capable of processing millions of bytes streaming from relatively highresolution video sources $(640 \times 480)$. A modestly appointed computer is thereby capable of completing both forwardand back-propagation steps on millisecond time scales. The ability to train and execute multi-billion-weight ANNs on digital computers has enabled nearly instantaneous translation of input camera frames from a robot -- for example, its forward view -- to navigational fields for the robot to follow toward pre-designated targets.

Creativity Machines are exemplified by ANN pairs. One side is pre-trained from sample data patterns embodying a targeted conceptual space. As it is internally or externally perturbed stochastically or systematically, this net generates output patterns representing potentially novel concepts or plans of action. The other side serves as a critic, selecting those ideas or strategies meeting criteria implicitly instilled within it via supervised learning. In the case of a control system, the perturbed net generates control strategies that are implemented upon approval by the critic net.

If a Creativity Machine is STANNO-based, it may cumulatively learn from its own mistakes, bootstrapping from no learning whatsoever, to progressively higher levels of competence.

Furthermore, if the critic is allowed to correct connection weights within the former net, it may react to inaccuracies therein by iteratively correcting connection weight values. In this way, the Creativity Machine concept leads directly to that of STANNOs.

The Creativity Machine itself absorbs knowledge of the problem space under consideration and then explores "confabulations" of the information to arrive at new possibilities for solutions. Through carefully controlled application of stimuli to the network, new and often remarkably unique results are output for consideration.

Figure 2 shows a diagram of one adaptation of STANNO technology. In this configuration, the feedback topology mentioned above is shown as part of the ANN module. The figure serves to illustrate core means by which these ANNs train themselves: human interactions in effect involve only instruction of the ANNs in their assigned objectives, relying upon the ANNs to perform the more detailed and intensive neural training much faster than possible through conventional means. The final trained configurations are derived by experimentation, evaluation of results, and updated programming of neural weights.

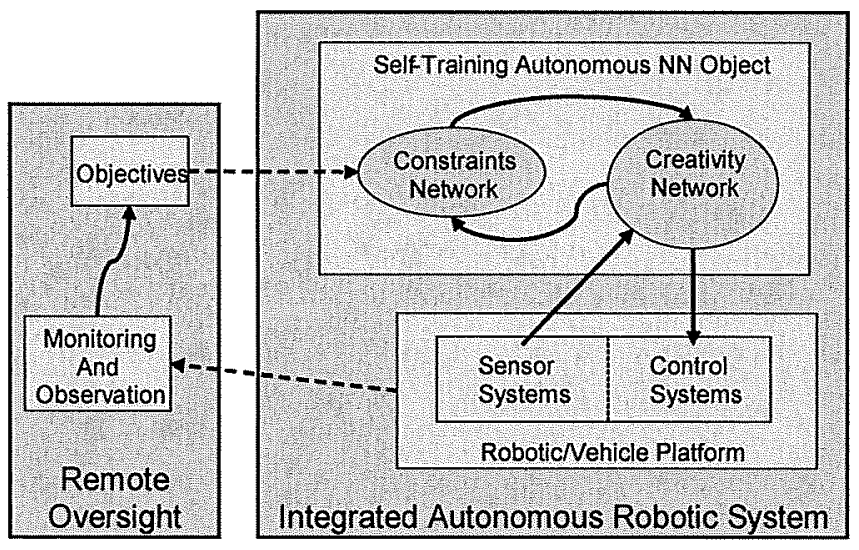

Figure 2: System Conceptual Diagram

As most people familiar with basic ANN theory know, when network weights are locked and cannot be further changed, the network is considered "static." Allowing weights to continue training during operation categorizes the network as "dynamic."

A very exciting aspect of this technology is that the ANNs not only learn behavior that can be creatively manipulated, but also learn constraints to be applied in an operational system. This contrasts very favorably with other techniques, including other ANN technologies and Genetic Algorithms, which require much fuller knowledge of the problem space's constraints prior to training or programming the intelligent core.

A very important possibility opens up to experimenters in view of this and the modular nature of STANNO technology. Consider making the Constraints Network static while allowing the Creativity Network to continue in dynamic operation. This scheme retains the benefits of dynamic network adaptation while keeping responses under fixed constraints. In fact, the Constraints Network can then potentially have standardized Independent Verification and Validation (IV\&V) applied in order to facilitate man-rating or other qualification. 


\section{EARLy Results}

In a set of impromptu experiments carried out on MSFC's Flat Floor during a preliminary visit in May 2006, IEI demonstrated basic capabilities of this technology in application to autonomous docking. The machine vision system utilized a Logitech webcam plugged into a laptop and taped to the top of the air sled. The image recognition package, developed for an entirely different purpose, consisted of a cascade of autonomously interconnecting STANNOs. This self-connecting ANN cascade was trained on four discreet images of the docking target from four evenly-spaced standoff ranges. Further, each of these images was approximately centered on the vertical midline of the target.

Video footage resulting from this exercise, illustrated in Figure 3, depicts the ANN's capability to not only identify cases in which white crosshairs generated in the images are nearly centered on the target, but also identify which range of distances to the target is most closely represented. Thus, the very rudimentary experiment shows how we may expect to estimate both alignment with and range to the target. The ability to form a crude navigational field useful for guiding the SAS is a result.

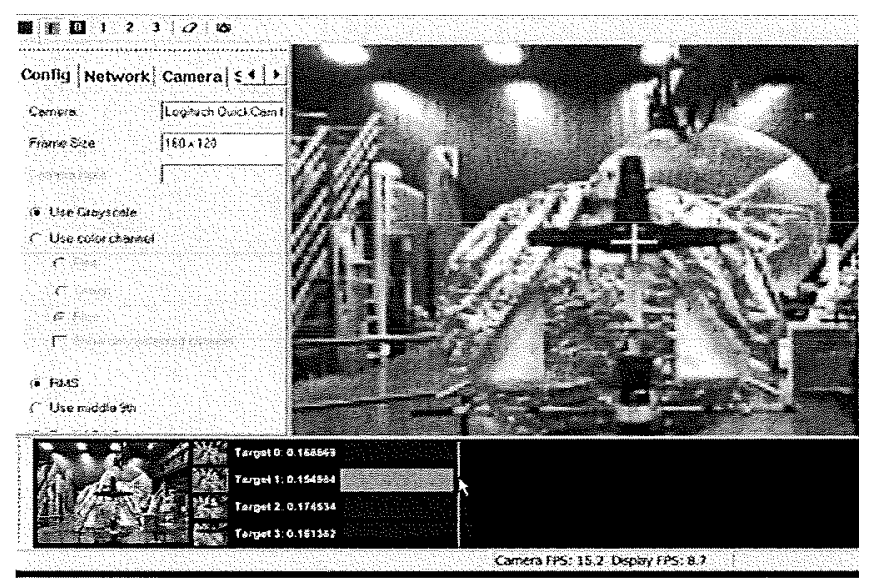

Figure 3: Early Impromptu Flat Floor Experimentation

It should be noted this demonstration was accomplished in less than an hour of actual time on the Flat Floor, and less than one minute total training of the ANN system to recognize the four images. Furthermore, we emphasize that the work was done without stereo imagery, with a commercial webcam purchased for less than one hundred dollars.

\section{First Series of Tests}

In early September 2006, IEI returned to MSFC for more testing on the Flat Floor. The first several days were spent making connection of the IEI laptop to the SAS control circuitry. Cabling was introduced to bypass the computer ordinarily utilized to command air thrusters on and off and maneuver the SAS.

The remainder of this visit was spent implementing schemes to adapt outputs from a self-connecting ANN cascade to the SAS. These ANNs generated a navigational field that would tend to rotate the SAS clockwise or counterclockwise in order to align with the target. In this case, thruster commands were coded directly in LabVIEW on the IEI laptop, in order to give researchers the chance to become more comfortable with the behavior of the air sled. Such caution seemed prudent because of restrictions on rates of SAS motion and general unfamiliarity of the researchers with the hardware. The ANNs were further trained to produce an attractive navigational field and command light forward thrusting in the event the SAS was aligned with the target. Finally, they were trained to produce a repulsive navigational field, resulting in light backward thrusting, to achieve station keeping just short of the target. Note that hard dockings were generally not a goal in this series of experiments.

After reasonable assurance of basic control and safety, a number of sequential runs with the SAS showed the STANNO cascade capable of accomplishing rendezvous with the target and initial action to take up station keeping at a range of about 3 meters. At this point it was discovered that because of the positions of thrusters selected for braking -- in this instance at the apex of the SAS triangle -- along with natural mismatching of individual thrust powers, braking caused the SAS to veer off-axis and break contact of the camera system with the target. Because searching for the target when out of view was not yet implemented, this generally terminated test runs.

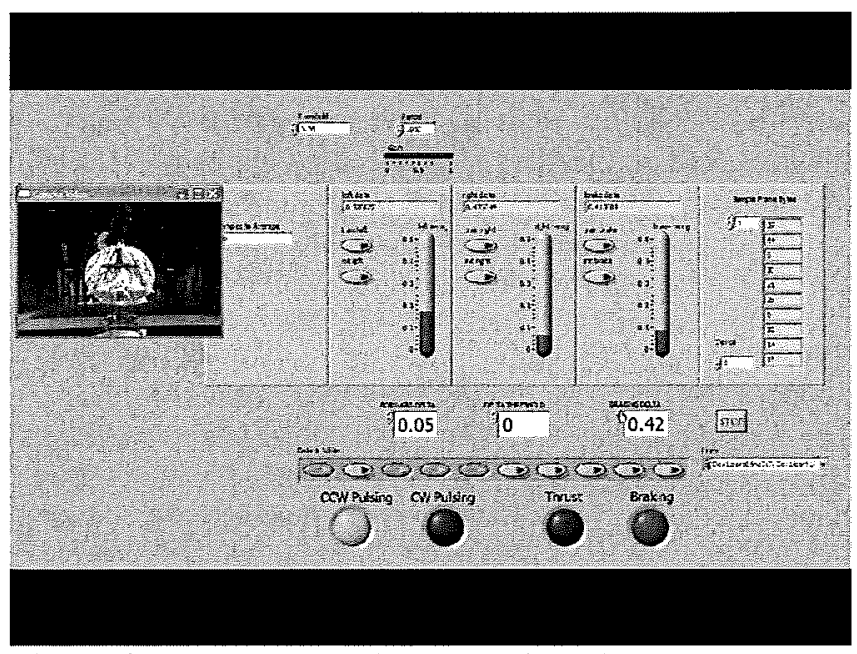

Figure 4: Front Panel Display during First Tests

Nevertheless, in several sequential tests the SAS came to satisfactory alignment and was under control at the station keeping point prior to brake firing, and in at least one case the system managed to recover and all but fully dock with the target; because no braking was implemented inside of the station keeping point, hard docking had to be prevented 
manually. Figure 4 shows the laptop display during this run. Note the small white crosshairs to either side of the central object in the video frame in the upper left; these mark the centers of interest of the ANNs, which served to cooperate in driving the navigational potential toward the docking fixture.

\section{SeCONd Series of TestS}

The next logical step was to give the STANNO cascade more direct control over SAS air thrusters, rather than coding their timing and duration patterns directly. Ultimately, the ANNs should be able to fire random thrust patterns, observe results, and rapidly develop control schemes necessary to dock with the target routinely and efficiently.

Incremental advancements would be made. Two different activities were undertaken to set the stage for accomplishment of these goals.

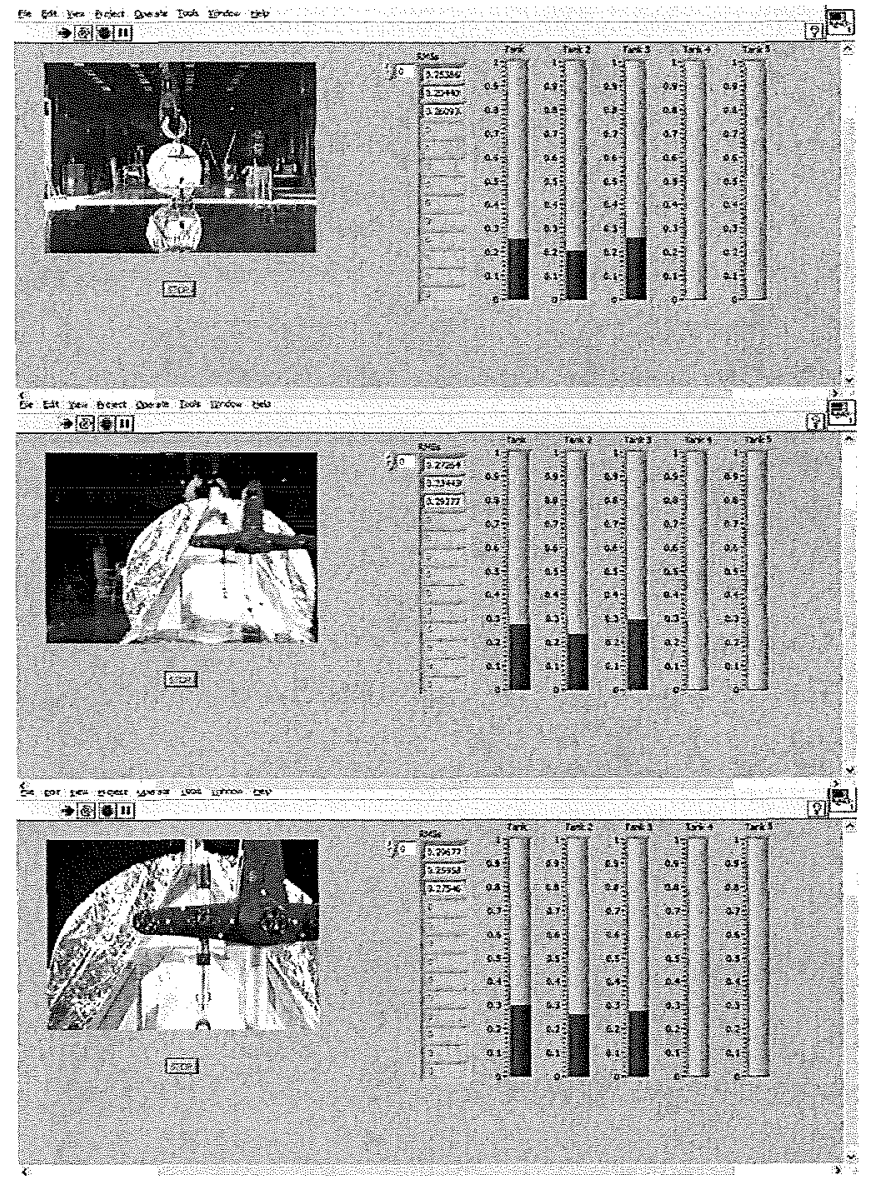

Figure 5: STANNO-based Navigation Field Generation

First, a video webcam was mounted and aligned to the front of the sled, as can be seen on close examination of Figure 1. Several frames of the target in different light conditions and from different perspectives were then presented to a STANNO cascade designed to now generate a navigational field or "channel" toward the docking target. Through prior offline training, this cascade became capable of converting, in real time, camera frames from the Logitech camera to a trough-like navigational field. This channel had a repulsive field that tended to restore the sled to the center of the trough, while constantly orienting it to face the target.

Next, a LabVIEW routine was developed to apply random thrusts on the SAS while collecting data on the its position and orientation within the STANNO-generated navigational field, as well as supplementary range, speed, and angular information gleaned directly from complementary sensor data.

These tests were delayed until December 2006, at which time this paper was overdue for publication. In the limited time available, it was only possible to demonstrate a reasonable semblance of ability to maintain forward motion of the sled within the navigation channel. However, because it was possible to produce only a small number of training exemplars for the neuro-control system, the sled tended to escape the navigational field.

Figure 5 through Figure 7 illustrate key results from these tests. The navigation channel is defined by blue level indicators to the right of each successive frame. Note that the actual point of interest on the target is no longer the very center of the black cross member, but in these tests is an optical target near the middle of the arm to the left.

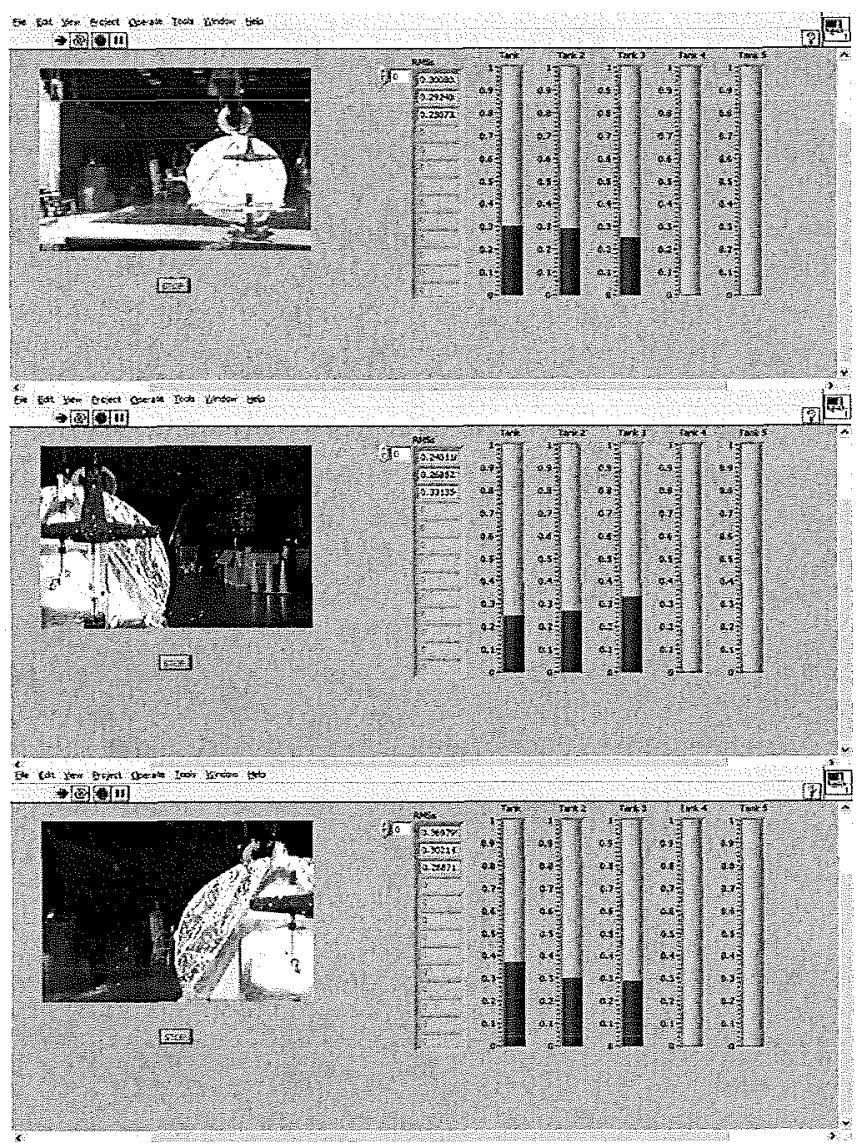

Figure 6: SAS Outside the Navigation Channel 
In Figure 5, as the sled travels down the center of the channel, the center blue indicator is lower than those to either side, as designed. Should the sled stray from this channel, the indicator pattern becomes asymmetric, as in Figure 6.

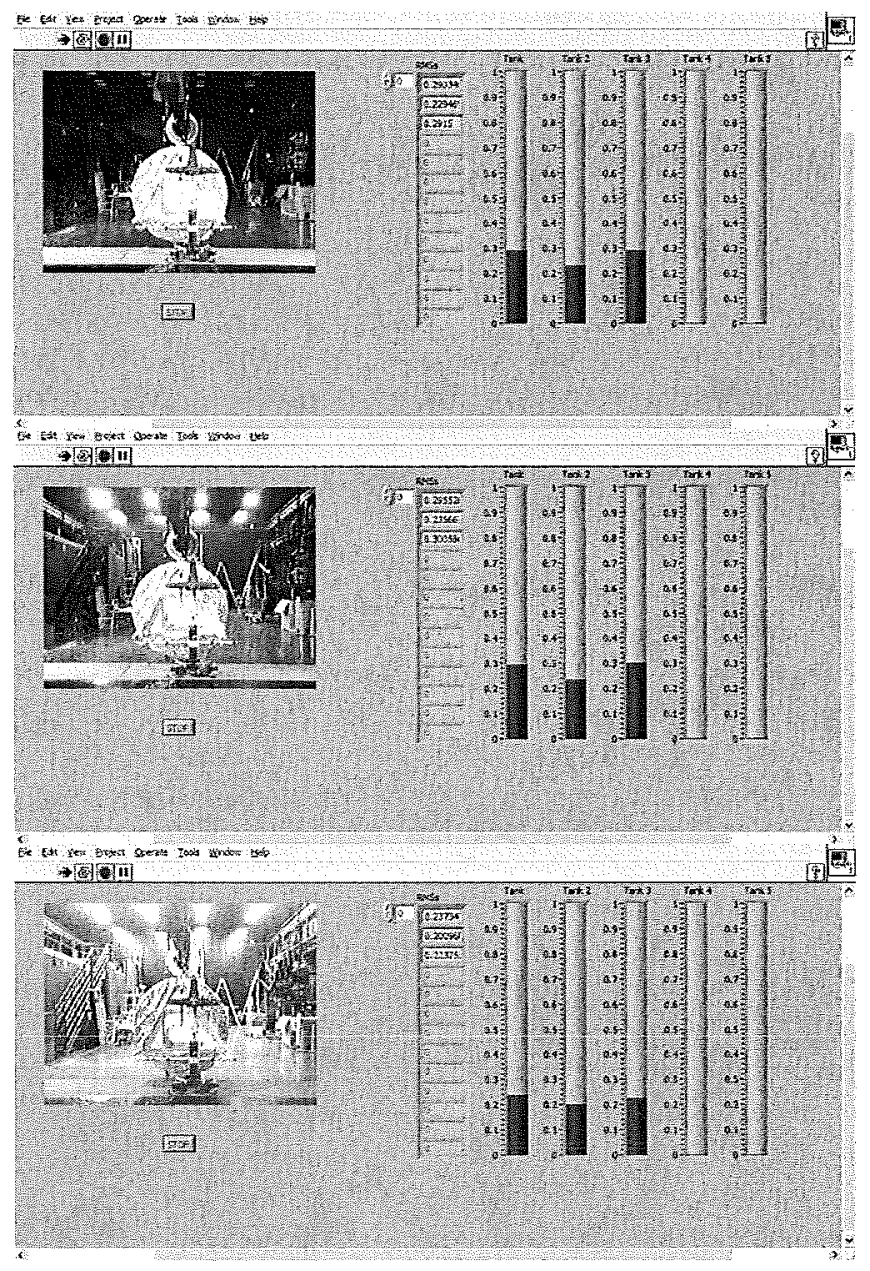

Figure 7: Channel Invariance with Illumination Change

Figure 7 illustrates the STANNO-generated navigation channel's relative immunity to variations in illumination. As ambient lighting is changed, the machine vision system correctly indicates the SAS is centered on the channel. In any of these lighting environments, deviation from the channel also produces appropriate asymmetric, repulsive fields consistent with the responses in Figure 6.

\section{Plans For Continued Work}

In coming months, the control system's training will be supplemented to better confine the SAS to the navigation channel.

Initially, this will mean generation of more and better training samples, along with some refinement of the ANNs' implementations, in order to more fully complete the objectives of the second set of tests.
The next step in planned work is to give STANNOs complete control over the air thrusters, with certain constraints in place for safety and efficacy. This will allow the ANNs to demonstrate ability to experimentally learn air sled operation and then autonomously control docking. In addition, it will allow the ANNs to learn multiple capabilities, including how to compensate for differences in thrust force from one air thruster to another, thrust pressures changing with supply and demand, unequal placement of thrusters, and imbalances in SAS load. Additionally, they should learn how to accomplish reasonably smooth and efficient motion overall. In fact, given enough time for the research, it would be productive to operate the ANNs dynamically and allow them to compensate for simulated thruster failures, shifts in sled load, and other changes in SAS handling characteristics.

Capacity for estimation of range will be calibrated against existing position-sensing instrumentation on the SAS, and then the ANNs will independently carry out a series of exercises designed to replicate standard Flat Floor docking work, but with only the benefit of video inputs.

First, the STANNO-controlled sled will carry out autonomous docking from an initial attitude with the docking target already in view and the air sled orthogonally aligned with the target.

Subsequent experiments will build upon these results, yielding capabilities not typically encountered in Flat Floor operations.

Continuing exercises will progressively show the ability of ANNs to successfully achieve autonomous dockings from non-orthogonal initial alignments and alignments which start without the target initially in view, or even with the target obscured by intervening objects.

To date, STANNOs have been realized primarily in software form, the most recent of which executes on a dualprocessor laptop computer. If the target system is too small to house and power the laptop, as is often the case with small robotic platforms in particular, interfaces are made by external umbilical or wireless connection.

This IR\&D projects as one product the realization of STANNOs and their adaptations in hardware form. At least initially, that form will most likely be grids of interconnected Field Programmable Gate Array (FPGA) devices. These grids are commonly called an "FPGA Fabric," as they knit together any number of FPGA devices, often along with other supporting electronics such as microprocessors, memory, and communication processors. Development of this capability is already underway.

Ultimately, it is expected that ANN processing will be done completely in hardware, making it possible not only to realize exceptionally dramatic increases in operational speeds, but also to implement more and larger networks. 
Eventual realization in ASIC or other forms is already under consideration.

\section{Conclusions}

Cumulatively over the course of this investigation, it has been shown that cascades of ANNs may be trained to generate navigational fields from raw camera frames. These ANN-generated navigational fields will potentially serve as virtual 'fly-through' channels to facilitate $A R \& D$ with space vehicles.

Preliminary data indicate that additional ANN cascades can learn to produce thrust sequences for aligning spacecraft with and propelling them through such navigational channels. Additional time at the Flat Floor or satisfactorily equivalent facilities will be required to train underlying ANNs and conduct more complete demonstrations.

\section{REFERENCES}

[1] Williamson, Marlin; Johnston, Nick; Howard, Richard; Hall, Drew; Gaines, Joe; and Chavis, Katherine S., "Automated Rendezvous and Docking Operations Evaluation," Proceedings of SPIE Defense and Security Symposium, Vol. 62210C, May 2006.

[2] Patrick, M. Clinton and Chavis, Katherine, NASA Marshall Space Flight Center 2006 Independent Research and Development program, Intelligent Vision Systems, project ID TA6-100.

[3] Thaler, S. L. (1997A), US Patent 5,656,666, Device for the autonomous generation of useful information, $8 / 19 / 1997$.

[4] Thaler, S. L. (1998), US Patent 5,845,271, Nonalgorithmically implemented artificial neural networks, 12/1/1998.

[5] Thaler, S. L. (1998), US patent 5,852,816, Neural network based database scanning system, 12/2/1998.

[6] Thaler, S. L. (1998), US Patent 5,852815, Neural network based prototyping system and method, 12/2/1998.

[7] Thaler, S. L. (2000), US Patent 6,014,653, Nonalgorithmically implemented artificial neural networks, $1 / 11 / 2000$.

[8] Thaler, S. L. (2000), US Patent 6.018,727, Nonalgorithmically implemented artificial neural networks, $1 / 11 / 2000$.

[9] Thaler, S. L. (2000), US Patent 6,115,701, Device for the autonomous generation of useful information, 9/5/2000.
[10] Thaler, S. L. (2000), US Patent 6,356,884, Device for the autonomous generation of useful information, 3/12/2002.

[11] Thaler, S. L. (1996) "Neural Networks that Create and Discover", PC AI, May / June, 1996

[12] Conrad, D.M, and Thaler, S. L. (1998) Real-Time Fault Detection Using Auto-associative Filtering, AIRTC, Oct. 1998.

[13] Thaler, S. L. (1995). "Virtual Input Phenomena" Within the Death of a Simple Pattern Associator, Neural Networks, 8(1), 55-65.

\section{ACKNOWLEDGEMENTS}

Special thanks are extended to Mr. Dean Vieau and Mr. Tom Tayon of IEI, and to Nick Johnston of NASA/MSFC, for invaluable assistance in carrying out tests.

\section{BIOGRAPHIES}

Clint Patrick is an Electronics Engineer in Advanced

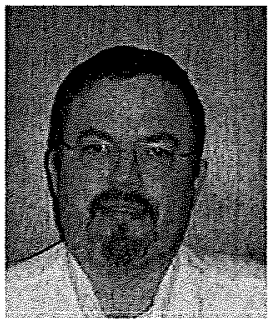
Avionics Sensors at MSFC. He has been with NASA more than over twenty-five years, working in electronic data systems; circuit design, layout, and production; FPGAs; system integration; system software development; spectroscopy; $A N N_{s}$; and parallel reconfigurable computing.

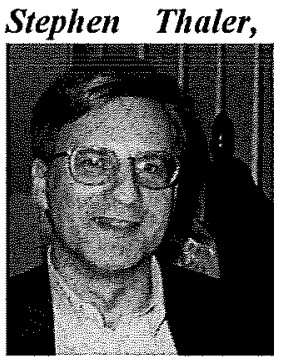

Ph.D., is President and CEO of Imagination Engines, Inc. He was formerly employed at both McDonnell Douglas Astronautics and Aircraft Companies where he engaged in nuclear space radiation hardening of EO systems, advanced low observables technology, and aircraftrelated sensing and control. In addition to many classified patents he holds through his work at $M D C$, he has privately amassed a significant suite of artificial neural network patents that enable many of the machine vision and neuro-control techniques implemented in this research.

Dr. Katherine Stevenson-Chavis is an Aerospace Engineer

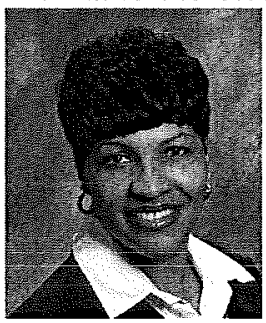
in Advanced Vehicle Sensors at MSFC. She has been with NASA for 2.5 years working with neural networks, data analysis, modeling and simulation, and fault diagnosis, isolation, and reconfiguration (FDIR). 